\title{
Dynamic Control of Autonomous Quadrotor Flight in an Estimated Wind Field
}

\author{
Nitin Sydney, Brendan Smyth, and Derek A. Paley
}

\begin{abstract}
We present a nonlinear, dynamic controller for a 6DOF quadrotor operating in an estimated, spatially varying, turbulent wind field. The quadrotor dynamics include the aerodynamic effects of drag, rotor blade flapping, and induced thrust due to translational velocity and external wind fields. To control the quadrotor we use a dynamic input/output feedback linearization controller that estimates a parametric model of the wind field using a recursive Bayesian filter. Each rotor experiences a possibly different wind field, which introduces moments that are accounted for in the controller and allows flight in wind fields that vary over the length of the vehicle. We add noise to the wind field in the form of Dryden turbulence to simulate the algorithm in two applications: autonomous ship landing and quadrotor proximity flight.
\end{abstract}

\section{INTRODUCTION}

In the past decade there has been growing interest in the use of unmanned aerial vehicles for both government [1] and commercial applications [2]. One of the concerns with small unmanned aerial vehicles such as quadrotors is their susceptibility to wind fields and gusts, which can not only degrade the performance of the vehicle but can also make them dangerous to operate in populated areas. Even without external wind gusts, quadrotors operating in proximity to one another are affected by the downwash from other quadrotors. In this paper, we present a strategy for controlling quadrotors in proximity to one another and/or in the presence of an estimated wind field. We illustrate the utility of our control strategy in two applications: autonomous control for landing on a ship and proximity flight of two quadrotors.

Many prior works in the area of quadrotor control, e.g., [3], [4], approximate the quadrotor dynamics by a linear system, for which standard linear controllers can be designed. More recent papers [5], [6], [7] use nonlinear control techniques like feedback linearization, backstepping, and sliding mode control. It is common for linear and nonlinear control techniques applied in the literature to use a six-degree-offreedom (6DOF) model for the quadrotor dynamics that neglects the effects of aerodynamic forces on the vehicle. Some notable exceptions ([8], [9]) model rotor effects such as blade flapping and induced thrust, which are important to the results in this paper. Other papers ([10], [11]) account for wind gust disturbances, but only as linear perturbations.

Nitin Sydney is a graduate student at the University of Maryland, College Park, Department of Aerospace Engineering,

Brendan Smyth is an undergraduate student at the University of Maryland, College Park, Department of Aerospace Engineering

Derek Paley is an Associate Professor at the University of Maryland, College Park, Department of Aerospace Engineering and Institute for Systems Research dpaley@umd. edu
In this paper, we develop a dynamic 6DOF model for a quadrotor that includes rotor blade flapping, induced thrust due to forward flight and climb, and aerodynamic drag. Unlike previous work, we allow the wind to vary from rotor to rotor, which is especially important for flight in close proximity to another quadrotor. A single rotor may lose thrust due to downwash impingement, causing a moment about the center of mass that can undesirably pitch and/or roll the vehicle. The model described here can also address wind fields in which the variation of the wind occurs at the length scale of the quadrotor $(\sim 0.5 \mathrm{~m})$. We design a layered feedback linearization controller to transform the quadrotor dynamics to a linear system, at which point standard regulation/tracking algorithms are used. The controller uses a Bayesian estimator that uses airspeed and position measurements (using, e.g., GPS and pitot tubes) to determine the parameters of an unknown wind field. The feedback linearization is split into an inner loop, for which the feedback linearization is exact, and an outer loop, for which we assume that the desired pitch and roll angles of the vehicle are small. We validate the algorithm by simulating proximity flight of two quadrotors and an autonomous ship landing, where the vehicle must travel in the turbulent air wake of a moving ship.

The contributions of this paper are (1) a 6DOF dynamic model for a quadrotor in wind that includes rotor blade flapping, induced thrust from translation, and aerodynamic drag; (2) a dynamic, feedback linearization controller that stabilizes a quadrotor in the presence of an unknown wind field. The controller incorporates a recursive Bayesian estimator that uses measurements of ground and air speed to provide an estimate of the wind field. One of the novel parts of the controller is that it treats the wind field on each rotor individually, so we can fly in wind fields that vary across the length of the quadrotor. The controller and estimator are validated in numerical simulations of reduced-order wind models with added (unmodeled) turbulence. Because of the virtues of the nonlinear control and nonlinear estimator design, we expect the control framework to have a variety applications to individual and multi-vehicle tasks in wind.

The rest of the paper is organized as follows. Section II discusses relevant background information, including feedback linearization, parametric wind field modeling of (turbulent) air wakes and rotor downwash, and recursive Bayesian estimation. Section III develops the 6DOF quadrotor dynamics, derives the feedback linearization controller, and presents the recursive Bayesian wind field estimator. Section IV presents simulation examples to demonstrate the control system. Section V summarizes the paper and ongoing work. 


\section{BACKGROUND INFORMATION}

\section{A. Feedback Linearization}

In this paper we use input/ouput feedback linearization of the nonlinear dynamics of the quadrotor [12]. Let $\mathrm{x} \in \mathbb{R}^{n}$ be the state vector of the quadrotor, $\mathbf{u} \in \mathbb{R}^{m}$ be the control, and $\mathbf{y} \in \mathbb{R}^{m}$ be the output. Consider (nonlinear) system dynamics of the form

$$
\begin{aligned}
\dot{\mathbf{x}} & =f(\mathbf{x})+\sum_{i=1}^{m} g_{i}(\mathbf{x}) u_{i} \\
\mathbf{y} & =\left[y_{1}, \ldots, y_{m}\right],
\end{aligned}
$$

where $f, g \in \mathbb{R}^{n}$. The feedback linearization technique described next allows us to design $\mathbf{u}$ to linearize (2) using state feedback control.

Let $r_{k}$ be the relative degree of the $k$ th output, where $k=1, \ldots, m$, and let $L_{f}^{k}\left(y_{i}(\mathbf{x})\right)$ denote the $k$ th Lie derivative of $y_{i}(\mathbf{x})$ with respect to $f[12]$. A control that linearizes the system (1)-(2) is [12]

$$
\mathbf{u}=\Delta^{-1}(\mathbf{x})(\boldsymbol{\nu}-b(\mathbf{x}))
$$

where

$$
\Delta_{i j}(\mathbf{x})=L_{g_{j}} L_{f}^{r_{i}-1} y_{i}(\mathbf{x}), \text { and } b_{i}(\mathbf{x})=L_{f}^{r_{i}-1} y_{i}(\mathbf{x}) .
$$

$\Delta(\mathbf{x})$ is the decoupling matrix for the system and the (linear) control $\nu$ can be designed to achieve the desired closed-loop behavior of the new system [12]

$$
\left[\begin{array}{c}
\frac{d^{r_{1}} y}{d t^{r_{1}}} \\
\vdots \\
\frac{d^{r_{m}} y}{d t^{r_{m}}}
\end{array}\right]=\left[\begin{array}{c}
\nu_{1} \\
\vdots \\
\nu_{m}
\end{array}\right] \text {. }
$$

Note that $\mathbf{u}$ requires the inverse $\Delta^{-1}(\mathbf{x})$ of the decoupling matrix, which means that $\Delta(\mathbf{x})$ must be invertible for all $\mathbf{x} \in \mathbb{R}^{n}$ in order for (1) to be feedback linearizable. Since our model quadrotor system does not meet this requirement, a two-layer feedback linearization approach is presented in Section III-B.

\section{B. Wind Field Model}

One of the novel contributions of this paper is the inclusion of aerodynamic effects in the feedback linearization controller, for which we need knowledge of any external wind fields or gusts. Since we may not know the precise wind field a priori, it is estimated online. In this section, we focus on parametric wind field models: first, a model for the air wake behind a ship that is a hybrid between potential flow and statistical turbulence; and second, a model for the downwash of a quadrotor helicopter. We estimate the wind parameters online using methods described in Section II-C.

We adopt an approach [13] to model a separated flow behind the ship using a complex potential in a simplified domain, called the $Z$-domain. The flow in the $Z$-domain contains elementary potentials, such as uniform flows, to represent ambient motion and vortices to represent separation.
This flow is transformed to the physical domain, called the $z$ domain, using a conformal mapping designed so that vortices in the $Z$-domain are placed after sharp bends or in notches in the $z$-domain. Note that without vortices, this technique would not produce separated flow in the $z$-domain.

Locations in the Z-domain are represented in complex coordinates as $Z=X+i Y$. Adding the potential of a vortex pair and a uniform flow yields

$\Omega(Z)=\frac{U_{\infty}}{2 \pi}\left\{Z-p-i \frac{(p+1)^{2}+s^{2}}{2 s} \log \left[\frac{Z-p+i s}{Z-p-i s}\right]\right\}$,

where $p \pm i s$ are the locations of the vortex centers. Since the vortices are of equal and opposite strength, there is a virtual surface on the real line through which flow cannot pass. The uniform flow represents the ambient flow and the vortex at $p+i s$ produces the separated flow in the $z$-domain.

Let $z=F(Z)$ be a conformal map from the $Z$-domain to the $z$-domain. The mapping $F(Z)$ takes the real line and shapes it to whatever the physical domain looks like. For example, for a backwards step, which is an approximation to the geometry of the deck of a ship suitable for landing an unmanned aerial system, the map $F(Z)$ is [13]

$$
z=\frac{h}{\pi}\left\{\sqrt{Z^{2}-1}+\log \left[Z+\sqrt{Z^{2}-1}\right]\right\} .
$$

From [13], we have that the magnitude $q$ and direction $\theta$ of the flow at location $Z$ are

$$
\begin{aligned}
& q=\operatorname{Re}\left\{\log \left[\frac{d \Omega}{d Z}\left(\frac{d F}{d Z}\right)^{-1}\right]\right\} \\
& \theta=-\operatorname{Im}\left\{\log \left[\frac{d \Omega}{d Z}\left(\frac{d F}{d Z}\right)^{-1}\right]\right\} .
\end{aligned}
$$

For the backwards step we get [13]

$$
\frac{d \Omega}{d z}=\frac{(Z-2 p-1) \sqrt{Z+1} \sqrt{Z-1}}{(Z-p+i s)(Z-p-i s)} .
$$

Using (7) and (9) the flow magnitude and direction are [13]

$$
\begin{gathered}
q=\sqrt{\frac{(X-2 p-1)^{2}+Y^{2}}{\left[(X-p)^{2}+(Y+s)^{2}\right]\left[(X-p)^{2}+(Y-s)^{2}\right]}} \times \\
\theta=\tan ^{-1}\left(\frac{Y+s}{X-p}\right)+\tan ^{-1}\left(\frac{Y-s}{X-p}\right)-\frac{1}{2} \tan ^{-1}\left(\frac{Y}{X-1}\right)+ \\
-\tan ^{-1}\left(\frac{Y}{X-2 p-1}\right)-\frac{1}{2} \tan ^{-1}\left(\frac{Y}{X+1}\right) .
\end{gathered}
$$

Figure 1 illustrates the flowfield in the $z$-domain generated by this transformation. Assuming that the complex plane represents the horizontal $(x)$ and vertical $(z)$ directions, the mean wind field is

$$
\begin{aligned}
& w_{x, m}=q \cos \theta \\
& w_{y, m}=0 \\
& w_{z, m}=q \sin \theta .
\end{aligned}
$$

For the application of proximity flight, we derive an expression for the downwash of a quadrotor based on [14] 


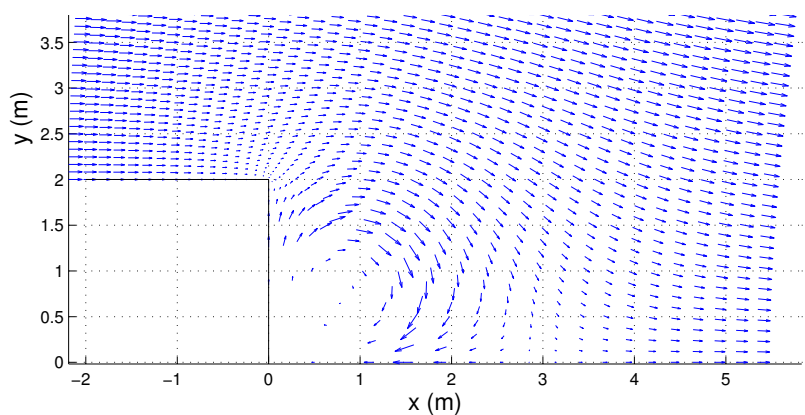

Fig. 1: Flowfield in the z-domain

momentum theory to describe the downwash of each rotor. Let $v_{i}$ be the induced velocity of a rotor, which is the vertical velocity directly underneath it. Then, the vertical velocity $w_{c}$ at any point $z$ below the rotor height $z_{r}$ is [14]

$$
w_{c}=v_{i}+v_{i} \tanh \left(-k \frac{z_{r}-z}{h}\right),
$$

where $k$ and $h$ are shaping parameters that control how rapidly the area of the streamtube below the rotor contracts to its steady-state value. Momentum theory [15] assumes that $w_{c}$ is uniform over the $x y$ plane for a given $z$ position. However, we know that the boundary of the downwash contracts as it speeds up, so we can identify the radial condition for when the vertical velocity goes to zero. Let $\rho$ be the radial distance of the downwash boundary from the rotor center $\left(x_{r}, y_{r}, z_{r}\right)$ and $R$ be the radius of the rotor. Using momentum theory [13]

$$
w_{z, m}= \begin{cases}w_{c} & \text { if } \rho<R / \sqrt{1+\tanh \left(-k \frac{z_{r}-z}{h}\right)} \\ 0 & \text { otherwise. }\end{cases}
$$

To get the full flowfield for all four rotors we combine the flowfield generated by all the rotors. Figure 2 shows an example of the quadrotor downwash with $v_{i}=4 \mathrm{~m} / \mathrm{s}$, where negligible or zero velocity vectors have been omitted.

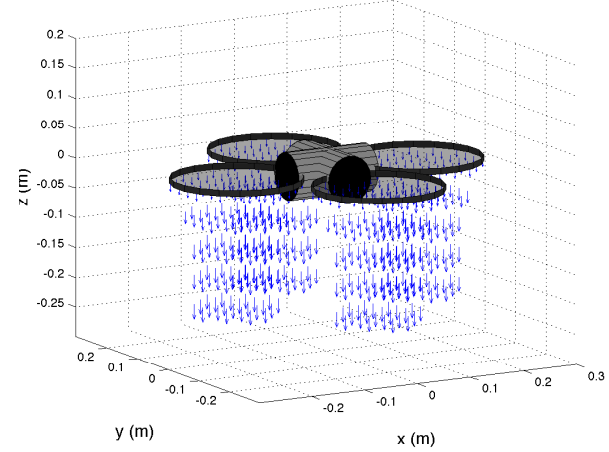

Fig. 2: Flow beneath a quadrotor using (16) as the downwash model.

Note that we assume that the downwash has no radial component of velocity. The justification for this assumption lies in the observation that the downwash tends to contract to its steady-state condition within 1-2 rotor radii, after which the flowfield is primarily vertical [15]. Thus, the model is valid only for proximity flight with separation greater than 1-2 rotor radii.

Since the wind fields described above are smooth, which is unrealistic for the air wake behind a ship or the downwash of a quadrotor, we add frozen (spatially constant) Dryden gust model to the mean wind field to simulate turbulence. The turbulent component of $w_{(\cdot)}$ is [16]

$$
w_{(\cdot), t}=\sum_{n=1}^{N} \sqrt{\Delta \omega \Phi\left(\omega_{n}\right)} \sin \left(\omega_{n} t+\phi_{(\cdot), n}\right),
$$

where $\omega_{n}$ is the frequency of the $n$th mode, $\Delta \omega$ is the spacing of the frequencies, $\phi_{(\cdot), n}$ is a random phase shift, and

$$
\Phi\left(\omega_{n}\right)=\sigma_{t}^{2} \frac{2 L_{t}}{\pi} \frac{1}{1+\left(L_{t} \omega_{n}\right)^{2}}
$$

is the power spectral density for a Dryden gust model [16]. Thus, the total wind field is

$$
w_{(\cdot)}=w_{(\cdot), m}+\sum_{n=1}^{N} \sqrt{\Delta \omega \Phi\left(\omega_{n}\right)} \sin \left(\omega_{n} t+\phi_{(\cdot), n}\right),(18)
$$

where the phase shifts are different for each spatial dimension.

\section{Recursive Bayesian Filtering}

With the wind fields described above, we implement a recursive Bayesian estimator to determine the field parameters. We assume that the wind field is parameterized by a set $\boldsymbol{\beta}$ of $P$ parameters. We seek an estimator that provides the best estimate $\hat{\boldsymbol{\beta}}$ given sensor measurements, such as airspeed, ground velocity, and attitude. The recursive Bayesian estimator is formulated as follows [17]. Let $\mathbf{z}\left(t_{k}\right)$ be the vector of noisy measurements at time $t_{k}$ from the sensors on the vehicle. The probability of $\boldsymbol{\beta}$ being the correct set of parameters at $t_{k}$ is [17]

$$
p\left(\boldsymbol{\beta} \mid \mathbf{z}\left(t_{1}\right), . ., \mathbf{z}\left(t_{k}\right)\right)=A p\left(\mathbf{z}\left(t_{k}\right) \mid \boldsymbol{\beta}\right) p\left(\boldsymbol{\beta} \mid \mathbf{z}\left(t_{1}\right), . ., \mathbf{z}\left(t_{k-1}\right)\right),
$$

where $p\left(\boldsymbol{\beta} \mid \mathbf{z}\left(t_{1}\right), . ., \mathbf{z}\left(t_{k}\right)\right)$ is the posterior probability density, $A$ normalizes the posterior to have unit integral, and $p\left(\mathbf{z}\left(t_{k}\right) \mid \boldsymbol{\beta}\right)$ is a conditional probability called the likelihood function, which represents the probability of receiving measurement $\mathbf{z}\left(t_{k}\right)$ given a set of parameters $\boldsymbol{\beta}$. The maximum likelihood estimate $\hat{\boldsymbol{\beta}}$ is the mode of (19) [17]. For multivehicle estimation with $N$ vehicles, the posterior due to measurements from all vehicles is obtained by

$$
p\left(\boldsymbol{\beta} \mid \overline{\mathbf{z}}\left(t_{k}\right)\right)=p\left(\boldsymbol{\beta} \mid \mathbf{z}_{1}\left(t_{1}\right), . ., \mathbf{z}_{n}\left(t_{k-1}\right)\right) \prod_{i=1}^{N} p\left(\mathbf{z}_{i}\left(t_{k}\right) \mid \boldsymbol{\beta}\right),
$$

where $p\left(\mathbf{z}_{i}\left(t_{k}\right) \mid \boldsymbol{\beta}\right)$ is the likelihood function for the $i$ th vehicle, $\mathbf{z}_{i}\left(t_{k}\right)$ is the measurement from the $i$ th vehicle at time $t_{k}$ and $\overline{\mathbf{z}}\left(t_{k}\right)$ is the measurements from all the vehicles up to and including time $t_{k}$. 


\section{DYNAMIC CONTROL OF A 6DOF QUADROTOR}

This section presents a nonlinear controller for a model quadrotor flying in an unknown, turbulent wind field. We start by deriving the dynamics of the vehicle in Section IIIA, where we include aerodynamic loads and perturbations not normally considered in the literature. In Section III-B we derive a layered feedback linearization controller that determines the thrust for each rotor. For this controller, we assume that the desired pitch and roll angles are small. In the final section, we present a Bayesian estimator that determines an estimate of an unknown wind field given (noisy) measurements of groundspeed, airspeed, and position of the vehicle.

\section{A. Quadrotor Dynamics}

The following 6DOF model for quadrotor flight in wind includes the effects of rotor blade flapping, induced thrust due to translational flight, and aerodynamic drag. Also novel is the individual treatment of blade flapping and induced thrust on each of the rotors, which is crucial to flight in the downwash of another vehicle or in the presence of a spatially varying flowfield.

The free-body diagram of the quadrotor in Figure 3 shows six forces acting on the quadrotor (gravity, aerodynamic drag, and the four thrust forces) and two reference frames (the inertial reference frame $\mathcal{I}$, which is centered at origin $O$ and has unit vectors $\mathbf{e}_{x}, \mathbf{e}_{y}$, and $\mathbf{e}_{z}$, and the body reference frame $\mathcal{B}$, centered at $G$ with unit vectors $\mathbf{b}_{x}, \mathbf{b}_{y}$, and $\mathbf{b}_{z}$ ). The inertial orientation of the body frame is described using a 3-2-1 Euler angle sequence of yaw $(\psi)$, pitch $(\theta)$, and roll $(\phi)$ angles.

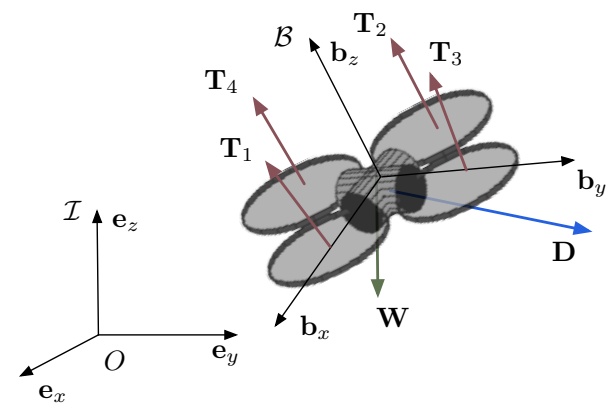

Fig. 3: Reference frames and free body diagram for quadrotor.

Figure 4 shows how the thrust vector $\mathbf{T}_{k}$ is deflected away from the $\mathbf{b}_{z}$ axis due to rotor blade flapping [15], which can be understood as follows. As the relative wind hits the rotor, the advancing side of the blade experiences increased flow and lift, whereas the retreating side sees decreased flow and lift, causing the blades to tilt due to the change in aerodynamic load. However, since the loading cycles occur at the same frequency as the rotation of the blade, there is a resonance effect that causes the maximum deflection to occur 90 degrees out of phase with the load location [15]. Thus, the rotor plane tilts away from the relative velocity vector, and redirects the thrust force.

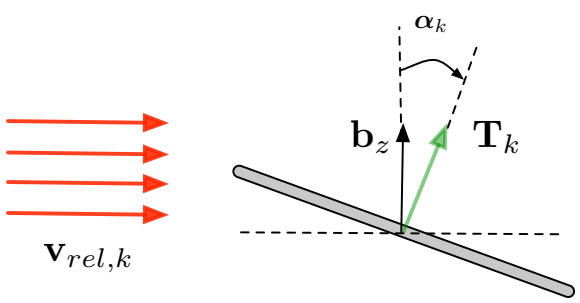

Fig. 4: Flap angle of the rotor due to a relative wind.

The air-relative velocity incident upon the $k$ th rotor is denoted $\mathbf{v}_{r e l, k}=u_{k} \mathbf{b}_{x}+v_{k} \mathbf{b}_{y}+w_{k} \mathbf{b}_{z}$. Blade flapping occurs due to the component of $\mathbf{v}_{r e l, k}$ in the body $x y$ plane [8]. Following [18], we make the approximation that the blade flap angle $\alpha_{k}$ is proportional to the magnitude of the velocity in the body $x y$ plane. Thus, $\alpha_{k}=k_{f} \sqrt{u^{2}+v^{2}}$, where $k_{f}$ is a proportionality constant that is common to all four rotors. To find $\mathbf{T}_{k}$, we use the geometry in Figure 4 with the flap angle $\alpha_{k}$ to determine

$$
\mathbf{T}_{k}=T_{k}\left(\bar{u}_{k} \sin \alpha_{k} \mathbf{b}_{x}+\bar{v}_{k} \sin \alpha_{k} \mathbf{b}_{y}+\cos \alpha_{k} \mathbf{b}_{z}\right),
$$

where $\bar{u}_{k} \triangleq u_{k} / \sqrt{u_{k}^{2}+v_{k}^{2}}, \bar{v}_{k} \triangleq v_{k} / \sqrt{u_{k}^{2}+v_{k}^{2}}$, and $T_{k}$ is the magnitude of the thrust of the $k$ th rotor.

In addition to the tilt of the thrust vector due to blade flapping, we also model the change in the magnitude of the thrust due to the relative wind velocity. There are two effects that cause this change [15]: the first is an increase in thrust due to horizontal translation, which is known as induced thrust; the second is a decrease in thrust if the component of relative wind in the $\mathbf{b}_{z}$ direction is negative. Essentially, the rotor loses thrust when it is in climb and gains thrust in horizontal translation. Note that we ignore the tilt in the rotor plane due to blade flap, which is justified because flap angles tend to be on the order of one degree in moderate relative wind [8]. Let $v_{h}$ be the induced velocity of the rotor in hover, which we assume is known (either using momentum theory or experimentally). The modified thrust can be calculated using [15]

$$
\begin{aligned}
v_{i, k} & =\frac{v_{h}^{2}}{\sqrt{u_{k}^{2}+v_{k}^{2}+\left(v_{i, k}+w_{k}\right)^{2}}} \quad \text { and } \\
T_{k} & =\frac{F_{k} v_{i, k}}{v_{i, k}+w_{k}},
\end{aligned}
$$

where $F_{k}$ is the thrust applied to the airmass for a given power (the control input) to the rotor. Equations (22)-(23) assume that the motor dynamics are sufficiently fast so that their transient behavior can be ignored. Note also that the equation for $v_{i, k}$ produces a fourth-order polynomial in induced velocity citehoffman. For the case of climb, there is only one positive root, which represents the physical solution for induced velocity and can be found numerically or analytically [15]. In hover, the induced velocity is $v_{h}$, whereas in descent the momentum theory solution above is not valid as the airflow through the rotor is not steady. 
An important limitation of the model (22)-(23) is that it only holds when $w_{k} \leq 0$ or $w_{k}>2\left|v_{h}\right|$ [8]; otherwise, the rotor is in Vortex Ring State (VRS), which is a region in which the aerodynamics are unsteady and momentum theory results due not apply. In practice, helicopters fly quickly through VRS to avoid any dynamic instabilities. It has also been shown that the thrust tends not to vary greatly in descent [8]. Ground effect is also not modeled by (22)-(23).

We assume that the drag force acts at the center of mass of the vehicle and hence causes no moments on the vehicle. The drag force is [8]

$$
\mathbf{D}=C_{d}\left\|\mathbf{v}_{G}\right\|^{2} \hat{\mathbf{v}}_{G}
$$

where $C_{D}$ is a drag coefficient and $\mathbf{v}_{G}=u_{G} \mathbf{b}_{x}+v_{G} \mathbf{b}_{y}+$ $w_{G} \mathbf{b}_{z}$ is the relative velocity at the center of mass (the hat ${ }^{\wedge}$ denotes a unit vector). Note that (24) is a pointparticle assumption for the drag force, which is a reasonable approximation since the other aerodynamic effects dominate the moments [8].

To get the equations of motion for the translational dynamics we express all of the forces in terms of the unit vectors in the inertial frame using the rotation matrix

$$
{ }^{\mathcal{I}} R^{\mathcal{B}}=\left[\begin{array}{ccc}
c \theta c \psi & s \phi s \theta c \psi-c \phi s \psi & c \phi s \theta c \psi+s \phi s \psi \\
c \theta s \psi & s \phi s \theta s \psi+c \phi c \psi & c \phi s \theta s \psi-s \phi c \psi \\
-s \theta & s \phi c \theta & c \phi c \theta
\end{array}\right] .
$$

Let

$$
\begin{aligned}
& C_{1}=\left(C_{D}\left\|\mathbf{v}_{r e l, C M}\right\| u_{C M}+\sum_{k=1}^{4} \bar{u}_{k} \sin \alpha_{k} \frac{F_{k} v_{i, k}}{v_{i, k}+w_{k}}\right) \\
& C_{2}=\left(C_{D}\left\|\mathbf{v}_{r e l, C M}\right\| v_{C M}+\sum_{k=1}^{4} \bar{v}_{k} \sin \alpha_{k} \frac{F_{k} v_{i, k}}{v_{i, k}+w_{k}}\right) \\
& C_{3}=\left(C_{D}\left\|\mathbf{v}_{r e l, C M}\right\| w_{C M}+\sum_{k=1}^{4} \cos \alpha_{k} \frac{F_{k} v_{i, k}}{v_{i, k}+w_{k}}\right) .
\end{aligned}
$$

Using (25) and (26)-(27), the equations of motion for translation expressed in the inertial frame are

$$
m\left[\begin{array}{c}
\ddot{x} \\
\ddot{y} \\
\ddot{z}
\end{array}\right]_{\mathcal{I}}={ }^{\mathcal{I}} R^{\mathcal{B}}\left[\begin{array}{c}
C_{1} \\
C_{2} \\
C_{3}
\end{array}\right]_{\mathcal{B}}-\left[\begin{array}{c}
0 \\
0 \\
m g
\end{array}\right]_{\mathcal{I}} .
$$

To determine the rotational dynamics we need the moments on the quadrotor caused by the forces in Figure 3. Gravity acts through the center of mass, so it does not contribute to the moments on the vehicle. (We also assume that the drag force acts through the center of mass, so it does not generate a moment.) Thus, the only moments are caused by the thrust forces and a yawing moment to counteract the rotor torques, such that the total angular momentum about the center of mass $G$ is conserved. Assume a symmetric geometry, where all the rotors are a horizontal distance $L$ and vertical distance $d$ from the center of mass, and that rotors 1 and 2 are situated along the $\mathbf{b}_{x}$ axis and rotors 3 and 4 are along the $\mathbf{b}_{y}$ axis. In order to balance the moment produced by spinning the rotors, assume that rotors 1 and 2 spin in the opposite direction of rotors 3 and 4 . Note that a controlled imbalance of the rotor moments is what achieves yaw rotation in a quadrotor.

Let $\boldsymbol{\omega} \triangleq p \mathbf{b}_{x}+q \mathbf{b}_{y}+r \mathbf{b}_{z}$ be the angular velocity of the quadrotor body frame with respect to the inertial frame. Let $I \triangleq \operatorname{diag}\left(I_{x}, I_{y}, I_{z}\right)$ is the moment of inertia matrix for the quadrotor and $M_{y}$ be a yawing moment produced by spinning the motors. (Note that the up-down symmetry of the quadrotor is sufficiently small enough that we can neglect the off diagonal terms of the moment of inertia matrix.) The rotational dynamics are

$$
I \dot{\boldsymbol{\omega}}=-\boldsymbol{\omega} \times I \boldsymbol{\omega}+\sum_{k=1}^{4} \mathbf{r}_{k / G} \times \mathbf{T}_{k}+M_{y}\left[\begin{array}{l}
0 \\
0 \\
1
\end{array}\right],
$$

where $\mathbf{r}_{k / G}$ is the distance vector from $G$ to the $k$ th rotor. By performing the requisite cross products, we arrive at the following rotational equations of motion:

$$
\begin{aligned}
I_{x} \dot{p} & =-\left(I_{z}-I_{y}\right) q r-\sum_{i=1}^{4} d \bar{v}_{k} \sin \alpha_{k} \frac{F_{k} v_{i, k}}{v_{i, k}+w_{k}} \\
& +L\left(\cos \alpha_{3} \frac{F_{3} v_{i, 3}}{v_{i, 3}+w_{3}}-\cos \alpha_{4} \frac{F_{4} v_{i, 4}}{v_{i, 4}+w_{4}}\right) \\
I_{y} \dot{q} & =-\left(I_{x}-I_{z}\right) r p-\sum_{i=1}^{4} d \bar{u} \sin \alpha_{k} \frac{F_{k} v_{i, k}}{v_{i, k}+w_{k}} \\
& +L\left(\cos \alpha_{2} \frac{F_{2} v_{i, 2}}{v_{i, 2}+w_{2}}-\cos \alpha_{1} \frac{F_{1} v_{i, 1}}{v_{i, 1}+w_{1}}\right) \\
I_{z} \dot{r} & =-\left(I_{y}-I_{x}\right) p q-L\left(\bar{v}_{1} \sin \alpha_{1} \frac{F_{1} v_{i, 1}}{v_{i, 1}+w_{1}}\right. \\
& \left.-\bar{v}_{2} \sin \alpha_{2} \frac{F_{2} v_{i, 2}}{v_{i, 2}+w_{2}}\right)-L\left(\bar{u}_{3} \sin \alpha_{3} \frac{F_{3} v_{i, 3}}{v_{i, 3}+w_{3}}\right. \\
& \left.-\bar{u}_{4} \sin \alpha_{4} \frac{F_{4} v_{i, 4}}{v_{i, 4}+w_{4}}\right)+c_{m} \sum_{k=1}^{4} F_{k},
\end{aligned}
$$

where $c_{m}$ is a constant that relates the applied thrust to the moment induced by spinning the rotor. In order to simulate the motion of the vehicle, we use the rotational kinematics for a 3-2-1 Euler angle sequence [19]:

$$
\begin{aligned}
\dot{\phi} & =p+q \sin \phi \tan \theta+r \cos \phi \tan \theta \\
\dot{\theta} & =q \cos \phi-r \sin \phi \\
\dot{\psi} & =q \sin \phi \sec \theta+r \cos \phi \sec \theta
\end{aligned}
$$

Equations (31)-(33) along with (29) fully describe the translational and rotational motion of the quadrotor. In the case of hover with no external wind field, these equations of motion reduce to the standard equations for a (rigid) quadrotor, e.g., [6] (omitted due to space constraints).

\section{B. Feedback Linearization Control}

We now derive an input/output feedback linearization of the quadrotor control system. First, the system is transformed to the form of (1) (omitted for space constraints) using $\mathrm{x} \triangleq$ 
$[x, y, z, \dot{x}, \dot{y}, \dot{z}, \phi, \theta, \psi, p, q, r]^{T}$ and $\mathbf{u} \triangleq\left[F_{1}, F_{2}, F_{3}, F_{4}\right]^{T}$. The outputs to be controlled are the $3 \mathrm{D}$ position and heading (yaw) of the vehicle, i.e., $\mathbf{y}=[x, y, z, \psi]^{T}$. A calculation of the relative degree [12] shows that each output has relative degree two (omitted for space constraints). Since the decoupling matrix $\Delta(\mathbf{x})$ defined in Section III-B is singular for this set of outputs, we cannot determine a control $\mathbf{u}$ to make the input-output system linear using position and heading as the outputs.

As an alternative, we first feedback linearize the system with output $\mathbf{y}_{i n}=[\phi, \theta, \psi, z]^{T}$, i.e., the attitude and altitude of the vehicle, and then feedback linearize the planar position. The first system represents the inner loop, whose inputs are the desired attitude and altitude. In this case, the relative degree for each output is two and the decoupling matrix $\Delta(\mathbf{x})$ is invertible everywhere except when $\phi=\pi / 2$ or $\theta=\pi / 2$. The feedback linearized system is $\ddot{\mathbf{y}}_{i n}=\boldsymbol{\nu}_{i n}$. We design $\boldsymbol{\nu}_{\text {in }}$ to stabilize the system to a desired altitude and (small) attitude $\mathbf{y}_{i n, d}=\left[\phi_{d}, \theta_{d}, \psi_{d}, z_{d}\right]^{T}$ and then transform $\nu_{i n}$ to $\mathbf{u}$ using (3).

The outer loop takes as input the desired (planar) position and yaw, and outputs the desired pitch and roll values to be used by the inner loop. We seek planar position dynamics of the form $\ddot{x}=\nu_{\text {out }_{1}}$ and $\ddot{y}=\nu_{\text {out }_{2}}$, where $\nu_{\text {out }}$ is the outer loop control. Let $\phi_{d}=\phi_{d}\left(\nu_{\text {out }_{1}}, \nu_{\text {out }_{2}}\right)$ and $\theta_{d}=$ $\theta_{d}\left(\nu_{\text {out }_{1}}, \nu_{\text {out }_{2}}\right)$. Assuming that the desired pitch $\theta_{d}$ and roll $\phi_{d}$ are small, the desired mapping (using (29)) is

$$
\begin{aligned}
\phi_{d} & =\left(\sin \psi \nu_{\text {out }_{1}}-\cos \psi \nu_{\text {out }_{2}}+C_{2}\right) / C_{3} \\
\theta_{d} & =\frac{\left(\cos \psi \nu_{\text {out }_{1}}+\sin \psi \nu_{\text {out }_{2}}-C_{1}\right)}{\left(C_{2} \sin \phi_{d}+C_{3} \cos \phi_{d}\right)} .
\end{aligned}
$$

The full control architecture is shown in Figure 5. The inputs to the closed-loop system are $x_{d}, y_{d}, z_{d}$, and $\psi_{d}$. Given $x_{d}$ and $y_{d}$ we calculate $\nu_{o u t}$, which are the (linear) control for the outer loop. Then we use $\nu_{\text {out }}$ to calculate $\phi_{d}$ and $\theta_{d}$ according to (37)-(38), which are used along with $z_{d}$ and $\psi_{d}$ to calculate the linear control $\nu_{i n}$ for the innertracking loop.

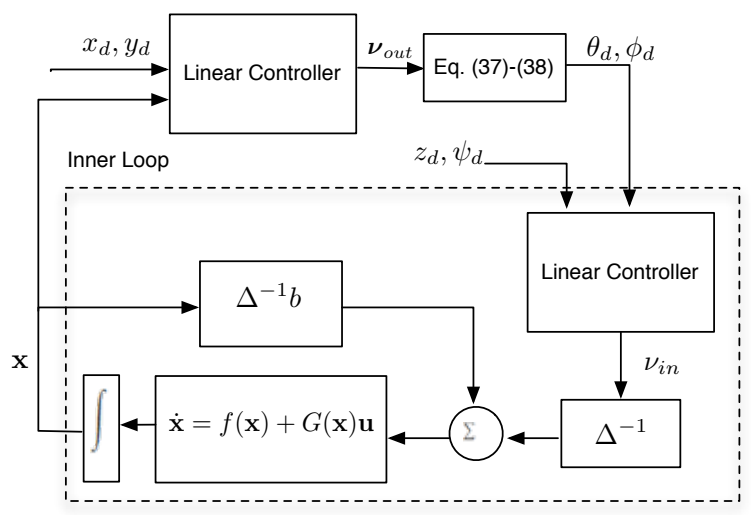

Fig. 5: Two-layer feedback linearization control system for 6DOF quadrotor.

The following two-vehicle simulation illustrates the benefit of accounting for the aerodynamics of the vehicle in the feedback linearization. The vehicles are commanded using
PID controllers on the inner and outer loops to a waypoint at the origin. Both vehicles start at the waypoint, and encounter a wind with a profile of $u=2 \mathrm{~m} / \mathrm{s}, v=2 \mathrm{~m} / \mathrm{s}$, and $w=-2(1-\cos (x)) \mathrm{m} / \mathrm{s}$. The first vehicle accounts for the aerodynamic loads, whereas the second vehicle does not, i.e., it neglects the flap angles, drag, and the thrust modification. Figure 6 shows that the vehicle accounting for the aerodynamics has a lower overshoot and a smaller settling time.
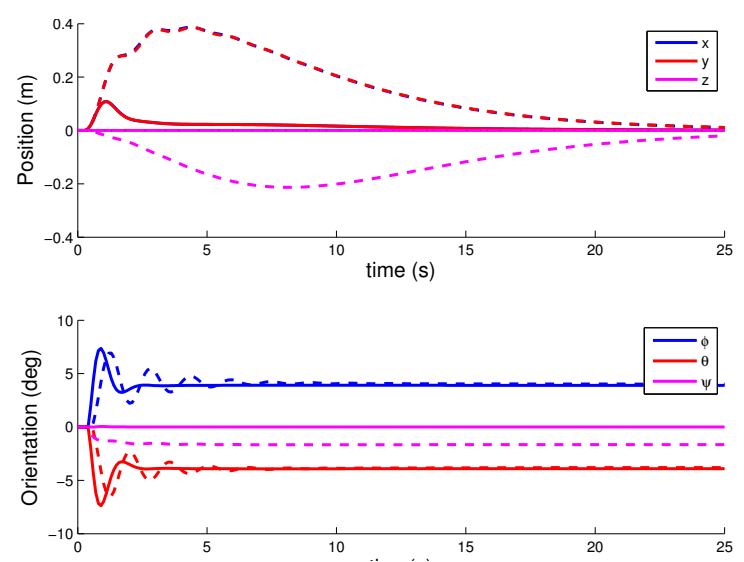

Fig. 6: Waypoint holding with (solid) and without (dashed) accounting for aerodynamic loads.

\section{Bayesian Estimation of Wind}

The control system described above allows us to stabilize a quadrotor in the presence of a known wind field; however, in general, this wind field will not be known. We now present a recursive Bayesian filter that produces an estimated wind field for use in the controller. To implement the recursive filter developed in Section II-C, we need a likelihood function, which relates the measurements to the wind and vehicle state. Assume the wind field is parameterized by parameter set $\boldsymbol{\beta}$, e.g. wind magnitude and direction for a uniform wind. Assume the ground velocity $\mathbf{v}=\dot{x} \mathbf{e}_{x}+\dot{y} \mathbf{e}_{y}+\dot{z} \mathbf{e}_{z}$ is available from GPS measurements (outdoors) or motion capture (indoors) and air velocity $\mathbf{v}_{\text {rel }}=u \mathbf{b}_{x}+v \mathbf{b}_{y}+w \mathbf{b}_{z}$ is measured by a pitot tube array (pitot tubes pointing along all axes so relative wind can be determined in each direction). (If the GPS updates are too slow for realtime control, a combination of GPS and an IMU could be used to determine position.) The expected air velocity measurement is

$$
\mathbf{v}_{r e l, e}(\boldsymbol{\beta}, \mathbf{v}) \triangleq\left[\begin{array}{c}
u_{e} \\
v_{e} \\
w_{e}
\end{array}\right]={ }^{\mathcal{I}} R^{\mathcal{B}}\left[\begin{array}{l}
w_{x, m}(\boldsymbol{\beta})-\dot{x} \\
w_{y, m}(\boldsymbol{\beta})-\dot{y} \\
w_{z, m}(\boldsymbol{\beta})-\dot{z}
\end{array}\right],
$$

where we have used the observation that the vehicle ground speed is the wind velocity plus the vehicle velocity relative to the wind. We assume that the pitot tubes are mounted orthogonally to the rotation of the vehicle such that vehicle rotation does not induce a velocity in the sensors. Using (39), we choose the following (Gaussian) likelihood function

$$
p\left(\mathbf{z}_{i}\left(t_{k}\right) \mid \boldsymbol{\beta}\right)=\exp \left[-\left(\mathbf{v}_{r e l}-\mathbf{v}_{r e l}\right)^{T}\left(\mathbf{v}_{r e l}-\mathbf{v}_{r e l, e}\right) / \sigma^{2}\right],
$$


where $\mathbf{z}_{i}\left(t_{k}\right)=\left[\begin{array}{llllll}\dot{x} & \dot{y} & \dot{z} & u & v & w\end{array}\right]^{T}$ and $\sigma^{2}$ represents measurement noise variance. This form for the likelihood function produces the desired behavior, namely that the likelihood increases when the parameters are close to the true value, and decreases when they are not.

As an example of using (40) and (20) to estimate a uniform wind, Figure 7 shows the estimator performance for a single quadrotor. In the simulation, the quadrotor is subjected to a uniform wind of $1 \mathrm{~m} / \mathrm{s}$ directed along the positive $y$ axis $(\pi / 2$ rad). Figure 7 shows the probability marginal distributions of wind speed (left) and direction (right) as measurements are incorporated in time. The dashed white line indicates the maximum likelihood estimate, which converges to the correct value, despite a noise level of $\sigma=0.1 \mathrm{~m} / \mathrm{s}$.
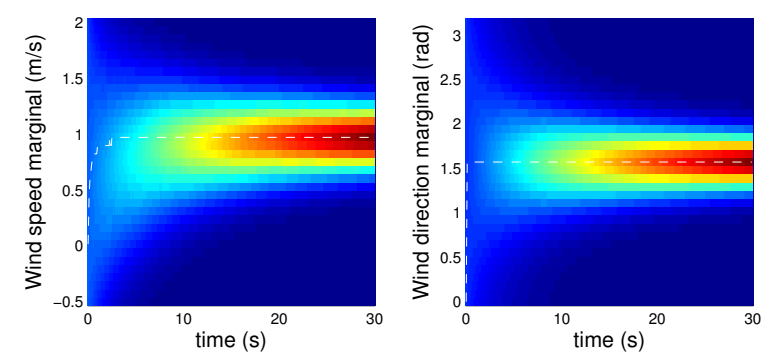

Fig. 7: Estimation marginals of wind speed (left) and direction (right).

\section{APPLICATION EXAMPLES}

In this section we apply the estimation and control technique developed in Section III to an autonomous ship landing and to quadrotor proximity flight.

\section{A. Autonomous Ship Landing}

The task of landing autonomously onboard a moving ship is made challenging by the separated flow that occurs on the deck of the ship, which can cause the autonomous vehicle to pitch and roll undesirably. The geometry of this situation is shown in Figure 8(a). Air flows over the forward section of the ship and then passes a sharp corner, where the flow separates and produces a vortex in the rear section of the ship where the quadrotor seeks to land. We use the potential flow methodology from Section II-B to generate the flowfield. The flowfield parameters $\boldsymbol{\beta}$ are the speed of the prevailing wind and the location of the vortex to determine the mean (nonuniform) wind field. As detailed in Section II-B, we add frozen Dryden turbulence to the mean wind field to simulate random eddies and flow fluctuations with a range of frequencies from 1-10 rad/s and randomly generated phase shifts.

In the simulation, the vehicle is commanded to go from $(x, y, z)=(4,0,2)$ to a landing location of $\left(x_{d}, y_{d}, z_{d}\right)=$ $(2,0,0)$. Figure 8 shows the controller performance. The colormap on the far right shows the estimation result for the wind speed. Observe that the vehicle converges to the desired landing location despite the unknown turbulence and the time it takes for the the parameter estimates to converge.

\section{B. Quadrotor Proximity Flight}

In this application we show how the dynamic controller in Section III can be used to fly one quadrotor directly underneath another, e.g., for formation flight. In the simulation, one quadrotor is commanded to hover at $(x, y, z)=(0,0,1)$ and a second quadrotor is commanded to fly underneath it to the origin. For comparison, we show a third (virtual) vehicle that does not compensate for the aerodynamics, i.e., the flap angles, drag coefficient, and climb velocity are all set to zero. In this simulation, $\boldsymbol{\beta}$ is the induced velocity of the hovering quadrotor. In addition, (frozen) Dryden turbulence affects all three vehicles. The results of the simulation are shown in Figure 9. The vehicle accounting for the aerodynamic loads settles at the appropriate location with no adverse affects, while estimating the induced velocity and shaping parameters of the downwash flowfield of the first vehicle (See Section IIB). The probability density is flat for the first three seconds before the quadrotor enters the downwash, then converges quickly once it enters the field. The third vehicle, however, experiences a sharp decent rate once it hits the flowfield as it has no knowledge of the wind (dashed lines in Figure 9(b)). In reality, this would likely put the rotors in VRS, which is not modeled here, and would cause the vehicle to become unstable.

\section{CONCLUSIONS AND ONGOING WORK}

This paper presents a control strategy for a 6DOF quadrotor in an estimated, spatially varying, turbulent wind field. The control strategy involves the use of a recursive Bayesian filter to estimate the wind field, which is incorporated into an input/output feedback linearization controller that includes aerodynamic effects on the vehicle such as blade flapping and drag. The novelty of the approach is that we formulate the dynamics to incorporate the effects of blade flapping, aerodynamic drag, and induced thrust in translation and due to external wind fields. We treat each rotor individually, so that the external wind can vary from rotor to rotor. We show that the inclusion of the flow compensation in the controller design improves the quadrotors ability to perform simple control objectives. We illustrate the dynamic control strategy in two applications: autonomous ship landing and proximity flight. The inclusion of the aerodynamics in the control scheme enables the stabilization of a quadrotor in the downwash of another quadrotor, even in the presence of unknown turbulence. In addition to the ongoing work mentioned in the paper, we are exploring more accurate methods for modeling the quadrotor downwash, including the effects of flow diffusion that occur downstream of the rotor and for motions other than hover and climb.

\section{ACKNOWLEDGEMENTS}

This material is based upon work supported by the U. S. Army under Grant No. W911W6112072.

\section{REFERENCES}

[1] A. Girard, A. Howell, and J. Hedrick. Border patrol and surveillance mission using multiple unmanned air vehicles. In Proc. of the 43rd IEEE Conf. on Decision and Control, pages 620-625, December 2004. 


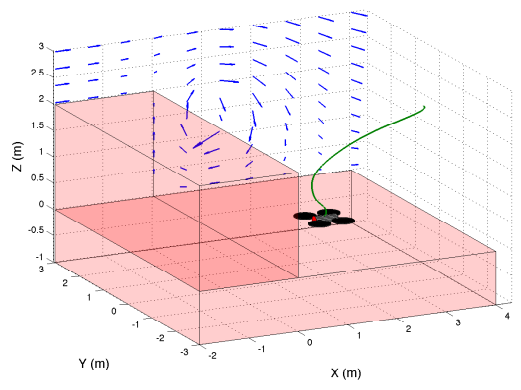

(a)

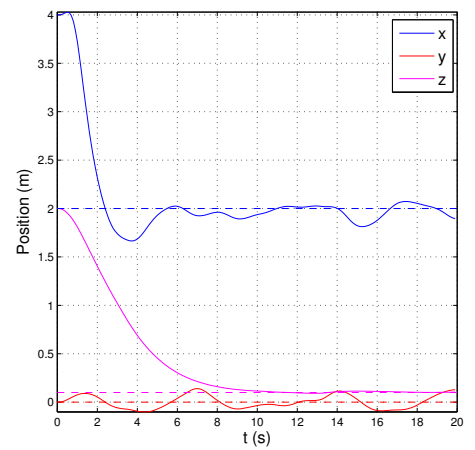

(b)

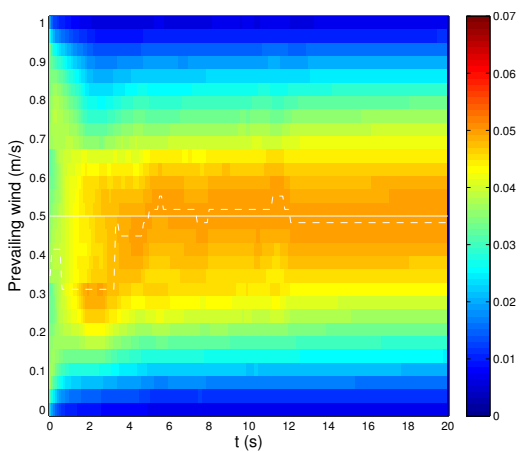

(c)

Fig. 8: Simulation of an autonomous ship landing with unknown turbulence. (a) The vehicle trajectory in 3D; (b) vehicle trajectories vs. time; (c) probability marginal for prevailing wind speed estimation.

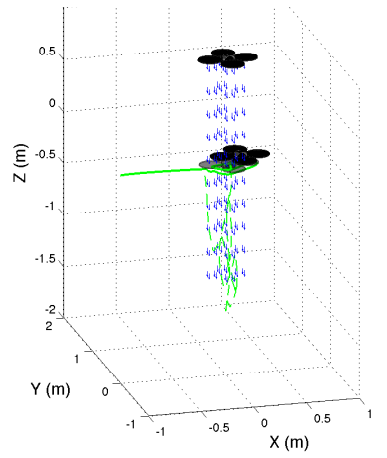

(a)

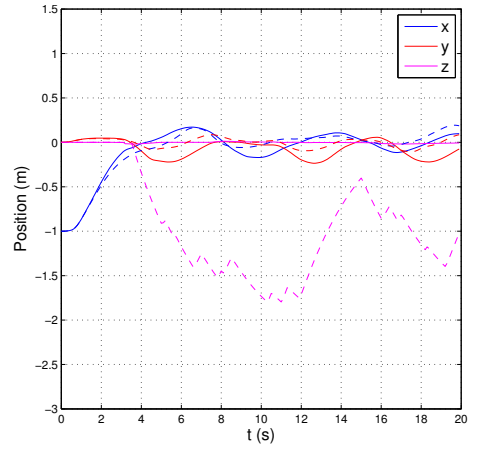

(b)

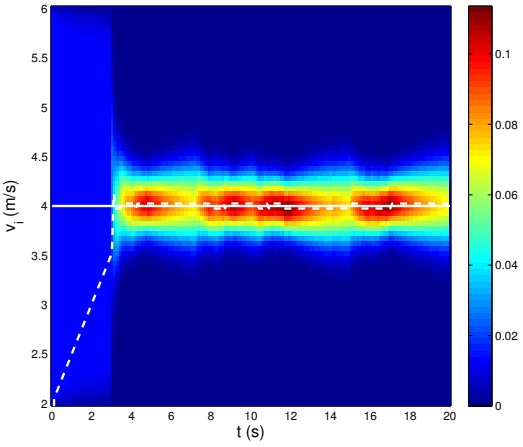

(c)

Fig. 9: Simulation of quadrotor proximity flight with unknown turbulence. (a) The vehicle trajectories in 3D with the shaded vehicle representing the quadrotor with no flow compensation; (b) vehicle trajectories vs. time; (c) probability marginal for induced velocity estimation.

[2] J.T.K. Ping, A. E. Ling, T. J. Quan, and C. Y. Dat. Generic unmanned aerial vehicle (UAV) for civilian application-a feasibility assessment and market survey on civilian application for aerial imaging. In 2012 IEEE Conf. on Sustainable Utilization and Development in Engineering and Technology, pages 289 -294, oct. 2012.

[3] M. Belkheiri, A. Rabhi, A. E. Hajjaji, and C. Pegard. Different linearization control techniques for a quadrotor system. In 2nd Int. Conf. on Communications, Computing and Control Applications (CCCA), 2012, pages $1-6$, Dec. 2012.

[4] B. Zhu and W. Huo. Trajectory linearization control for a quadrotor helicopter. In 8th IEEE Int. Conf. on Control and Automation (ICCA), 2010, pages $34-39$, June 2010.

[5] D. Mellinger and V. Kumar. Minimum snap trajectory generation and control for quadrotors. In IEEE Int. Conf. on Robotics and Automation (ICRA), 2011, pages 2520 -2525, may 2011.

[6] A. Benallegue, A. Mokhtari, and L. Fridman. Feedback linearization and high order sliding mode observer for a quadrotor UAV. In Int. Workshop on Variable Structure Systems, 2006. VSS'06., pages 365 -372 , June 2006.

[7] E. de Vries and K. Subbarao. Backstepping based nested multi-loop control laws for a quadrotor. In 11th Int. Conf. on Control Automation Robotics Vision (ICARCV), 2010, pages 1911 -1916, Dec. 2010.

[8] G. Hoffmann, H. Huang, S. Waslander, and C. Tomlin. Quadrotor Helicopter Flight Dynamics and Control: Theory and Experiment. AIAA, 2007.

[9] C. Powers, D. Mellinger, A. Kushleyev, B. Kothmann, and V. Kumar. Influence of aerodynamics and proximity effects in quadrotor flight.
In Proc. of the Int. Symp. on Experimental Robotics, June 2012.

[10] K. Alexis, G. Nikolakopoulos, and A. Tzes. Experimental model predictive attitude tracking control of a quadrotor helicopter subject to wind-gusts. In 18th Mediterranean Conf. on Control Automation (MED), 2010, pages $1461-1466$, June 2010.

[11] K. Alexis, G. Nikolakopoulos, and A. Tzes. Constrained-control of a quadrotor helicopter for trajectory tracking under wind-gust disturbances. In MELECON 2010, pages 1411 -1416, April 2010.

[12] H. K. Khalil. Nonlinear Systems. Prentice Hall, 3rd edition, 2002.

[13] A. Verhoff. Two-dimensional potential flow solutions with separation. Journal of Fluid Mechanics, 657:238-264, 2010.

[14] J. Seddon and S. Newman. Basic Helicopter Aerodynamics. AIAA, 3rd edition, 2011.

[15] J. G. Leishman. Principles of Helicopter Aerodynamics. Cambridge University Press, New York, 2000.

[16] F. Hoblit. Gust Loads on Aircraft: Concepts and Applications. AIAA Education Series, 1998.

[17] N.J. Gordon, D. J. Salmond, and A.F. Smith. Novel approach to nonlinear/non-Gaussian Bayesian state estimation. In Radar and Signal Processing, volume 140, pages 107-113, 1993.

[18] H. Huang, G. M. Hoffmann, S. L. Waslander., and C. J. Tomlin. Aerodynamics and control of autonomous quadrotor helicopters in aggressive maneuvering. In IEEE Int. Conf. on Robotics and Automation, 2009. ICRA '09., pages 3277 -3282, May 2009.

[19] J. Kasdin and D. Paley. Engineering Dynamics: A Comprehensive Introduction. Princeton University Press, 2011. 